
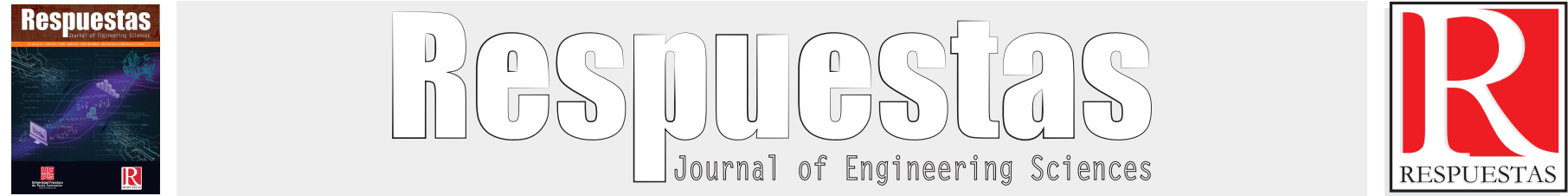

Artículo Original

https://doi.org/10.22463/0122820X.1613

\title{
Evaluación financiera para la implementación de la semimecanización del sistema de transporte interno de la mina Shipio, 2017
}

Financial evaluation for the implementation of the semimechanization of the internal transport system of the Shipio mine, 2017

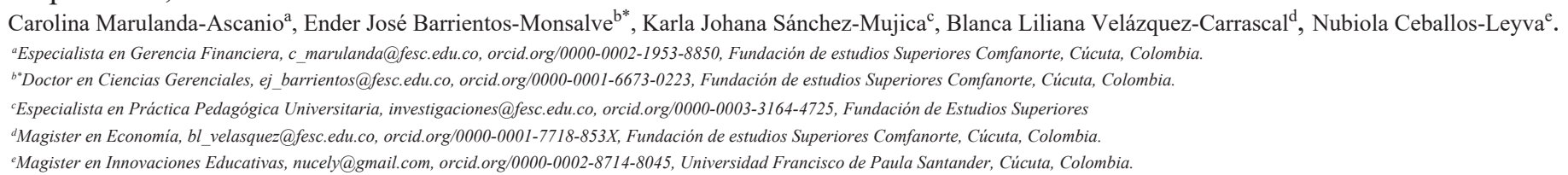
'Magister en Innovaciones Educativas,nucely@gmail.com,orcid.org/0000-0002-8714-8045,Universidad Francisco de Paula Santander.Cúcuta,Colombia.

Cómo citar: C. Marulanda-Ascanio, E.J. Barrientos-Monsalve, K.J. Sánchez-Mujica, B.L. Velázquez-Carrascal y N. Ceballos-Leyva. "Evaluación financiera para la implementación de la semimecanización del sistema de transporte interno de la mina Shipio, 2017", Respuestas, vol. 23 , no. S1, pp. 119 - 126, 2018. https://doi.org/10.22463/0122820X.1613

Recibido: Febrero 29, 2018; Aprobado: Julio 28, 2018.

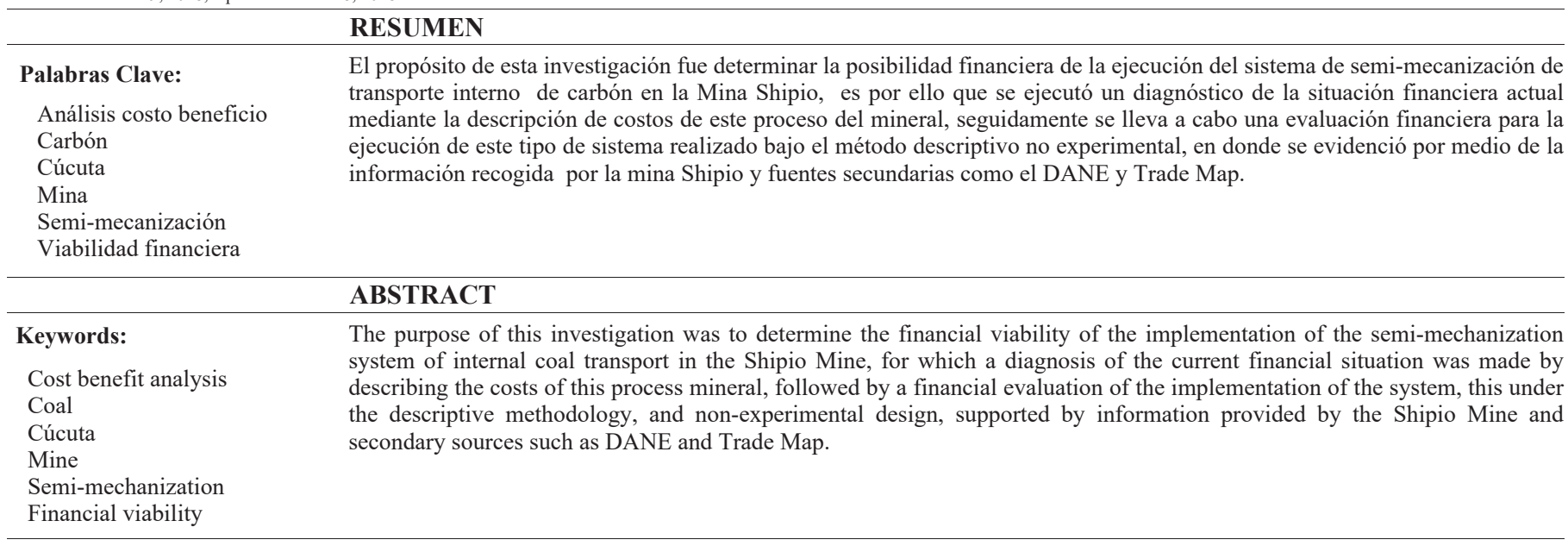

\section{Introducción}

El carbón constituye una de las fuentes de energía más importantes del mundo, y su importancia en el contexto mundial sigue en su alto nivel por la importancia en estos contextos, por su parte, [1] El carbón es uno de los principales generadores de energía más importante del mundo. Esto lo confirman cifras de exportación a nivel mundial tomadas del portal Trade Map, las cuales muestran que en el año 2006 se exportaron 49.747.351 miles de dólares de hullas y para el 2014 esta cifra ascendió a 97.480.260 miles de dólares.

De esta manera se evidencia la importancia de este contexto por cuanto la generación de energía de este elemento sigue en auge, aumentando las cifras de exportación a nivel mundial. Uno de los países latinoamericanos que exporta este mineral es Colombia y la la Agencia Nacional de Minería (ANM) estableció que la producción de carbón en Colombia para el año 2016 fue de 90.511 .989 toneladas de carbón, ubicándolo en el quinto puesto dentro de los principales exportadores de éste en el mundo, con 4.392.669 miles de dólares FOB en Hullas en el año 2016; en cuanto a Coques, exportó para el mismo año 246.765 miles de dólares FOB. A su vez, Colombia es uno de los países de la región latinoamericana contando con unos aproximados 16.992 millones de toneladas en donde los cuales 7.063 son medidas, 4571 son indicadas, 4.237 son inferidas y 1.119 son recursos hipotéticos [2].

Teniendo en cuenta lo anterior, Colombia posee un potencial tanto en recursos como en exportaciones, es por esto que la Agenda interna para la productividad y la competitividad de Norte de Santander incluye dentro del sector minero la apuesta productiva por el carbón, para lograr incrementos en la producción y ampliación de la oferta en el Departamento. "Norte de Santander posee ventajas competitivas en la producción de carbón térmico, pues los yacimientos de la región se distinguen por el alto poder calorífico y la baja emisión de cenizas y azufre. Es un carbón destinado principalmente a las centrales térmicas y la industria metalúrgica. Sin embargo, el mejora-

*Autor de correspondencia. 
miento en la infraestructura de las vías del transporte del carbón es indispensable para el desarrollo de esta actividad en la medida en que reduciría los costos de transporte [3].

Por otra parte, los actuales sistemas de transporte interno de la minería subterránea de carbón de pequeña y mediana escala implementada en Norte de Santander, se caracterizan según la misma fuente anterior. Por el mediano grado de tecnificación que involucra tecnología y control del impacto ambiental con inversiones tecnológicas de menor proporción, sistemas que restan ventajas competitivas al sector al incurrir en altos costos de producción, esto para mejorar las gestiones en las organizaciones [4]. Y es por ello donde se debe lograr aspectos positivos en la organización a fin e hacer más eficiente todas las gestiones y con resultados óptimos.

El carbón que produce la empresa Carbones de Toledo S.A., es de tipo Térmico, cuyas características de calidad, le abren un importante mercado en la comercialización local, abarcando una dinámica de interacción entre las empresas [5]. Tanto en el mercado del carbón térmico tipo exportación crudo, como en la venta de carbón para consumo en las plantas generadoras de electricidad TERMOTASAJERO S.A. E.S.P de la región. La demanda de este tipo de material es apreciable si se tiene en cuenta que la producción anual de las minas se vende sin ningún traumatismo ya que se cuenta con un contrato a largo plazo de suministro de carbón para la empresa TERMOTASAJERO S.A. E.S.P (ver tabla 1), a pesar de esto la empresa Carbones de Toledo S.A, tiene como objetivo a mediano plazo obtener otras contracciones del mercado; ya que la calidad del carbón explotado en cuanto a su calidad en BTU superiores a los $7250 \mathrm{kcal} / \mathrm{Kg}$, cenizas bajas menores al 10\%, azufre menor del 1\%, materia volátil media, y niveles de fósforos bastante bajos, hacen que los consumidores internos de la región demanden el carbón térmico que se produce en los títulos mineros.

Sin embargo, la producción actual anual de la empresa en el contracto de la mina el SHIPIO, no ha logrado sobrepasar las 60.000 toneladas/ anuales, lo que le impide a la empresa realizar negocios importantes con otros consumidores de la región y del exterior, y buscar mayores beneficios.

\section{Objetivo general}

Generar una evaluación financiera para la implementación de la semimecanización del sistema de transporte interno de la mina Shipio, 2017

\section{Objetivos específicos}

Explorar la situación actual sobre las finanzas para la implementación de la semimecanización del sistema de transporte interno de la mina Shipio, 2017.

Analizar los contextos financieros para la implementación de la semimecanización del sistema de transporte interno de la mina Shipio, 2017

Concebir una evaluación financiera para la implementación de la semimecanización del sistema de transporte interno de la mina Shipio, 2017

\section{Marco Teórico}

\section{Evaluación Financiera 1.1.}

Dentro del contexto de la presente investigación se sustentó por medio de autores a fin de ver los escenarios que se estudiaron en este trabajo. [6] La Evaluación Financiera es un proceso financiero mediante el cual una vez conocida la inversión inicial (inversión requerida para que el proyecto entre en operación) y los beneficios netos esperados, tiene como propósito principal medir la rentabilidad de un proyecto de inversión, Es por ello que el presente trabajo presenta dicho análisis a fin de mostrar los beneficios y la necesidad de la implementación del sistema de semimecanización del transporte interno de la mina. De la misma manera el autor señala que el fracaso de los negocios obedece a la improvisación de sus procesos y cuando no se sustenta en estudios confiables la inversión estará en riesgo, de esta manera estos estudios financieros minimizan al máximo los riesgos que puedan aparecer en el futuro en estas organizaciones, [7] y más aún en un mundo altamente complejo donde se debe estar cuidando los detalles de todas las organizaciones, [8] y más dentro de una economía emergente como la Colombiana.

En este mismo orden de ideas para, [1] Con el estudio técnico se pretende verificar la posibilidad técnica de fabricación del producto, o producción del servicio, para lograr los objetivos del proyecto. El objetivo principal de este estudio es determinar si es posible lograr producir y vender el producto o servicio con la calidad, cantidad y costo requerido; para ello es necesario identificar tecnologías, maquinarias, equipos, insumos, materias primas, procesos, recursos humanos, con esto se prevé que la inversión con base a la propuesta de la semimecanización pueda ser factible dentro de los contextos de la mina señalada. Así mismo el autor señala que los proyectos deben tener un ciclo de vida iniciando y terminando su proceso, esto por medio de una serie de etapas que comprenden el cumplimiento de los objetivos establecidos tratando de reducir los riesgos establecidos así como también las incertidumbres y esto se debe realizar desde la concepción o idea del proyecto hasta que se cumpla su materialización y dejar suficientes elementos para seguir avanzando en ello.

\section{Generalidades}

Localización Del Proyecto: El área de contrato N CL6-081 se encuentra ubicada en el departamento de Norte de Santander, en la jurisdicción del municipio de Cúcuta en la vereda Carri11 . 
Tabla I. Ubicación del Contrato CL6-081

\begin{tabular}{|c|c|c|}
\hline Vereda & Municipio & Departamento \\
\hline Carrillo & Cúcuta & Norte de Santander \\
\hline
\end{tabular}

El área objeto del contrato minero de la mina El Shipio, se encuentra descrita así: Punto arcifinio (P.A.), dado por la intersección de la quebrada vieja Felisa con el rio Pamplonita, de acuerdo al Instituto Geográfico Agustín Codazzi (IGAC), el área se encuentra ubicada dentro de la plancha topográfica No. 99-I-B, el titulo corresponde a una extensión superficiaria total de 647 Hectáreas y $9468 \mathrm{~m} 2$ establecido en una zona determinada por la por los puntos, rumbos, distancias y coordenadas de la siguiente alinderación, definida por las coordenadas planas origen Bogotá. Ver Tabla III.

\section{Aspectos Técnicos De La Explotación Minera}

Labores Mineras: El área del contrato la empresa cuenta con tres montajes operativos independientes denominados Shipio 1 - 2 e Inclinado 1, el montaje denominado Inclinado 1 está inactivo.

Acceso y Desarrollo: es el túnel o galería principal por medio del cual se accede al yacimiento, y se realice el avance y sostenimiento continúo del mismo, durante toda la vida útil del proyecto hasta agotar reservas.

Shipio 1: Se avanzó una cruzada a partir de la cota 791.54 msnm en roca con sección de 2,8 a 3,2 m2 y dirección $\mathrm{N} 56^{\circ} \mathrm{W}$ de una longitud de 450 metros. El resumen de la cruzada se presenta a continuación:

En la actualidad la cruzada de shipio 2 sirve de soporte técnico para el circuito de ventilación de la mina, ya que a través del nivel 1 del manto 5 se comunica con la cruzada de shipio 2; a través de esta labor de desarrollo se evacuan los estériles de la mina y se disponen en el botadero localizado en las proximidades de la cruzada de shipio 1 , y por esta cruzada y el nivel del manto 5 está instalada la infraestructura de la red neumática para soporte de la mina.

El desagüe de la mina se realiza por la cruzada 1 en su totalidad.

Shipio 2: Se avanzó una cruzada a partir de la cota 799.34 msnm en roca con sección de 3,2 a 3,6 m2 y dirección $\mathrm{N} 79^{\circ} \mathrm{W}$ de una longitud de 448 metros. El resumen de la cruzada se presenta a continuación:

Todas las labores de acceso y desarrollo cuentan con carrilera para el transporte del estéril y del mineral y cuneta para la evacuación de los niveles de agua interceptados.
Actualmente la Mina Shipio cuenta con la distribución del personal en sus diferentes etapas de la siguiente manera:

El actual mecanismo de transporte interno de minería subterránea consiste en un coche o vagoneta de capacidad de una tonelada (1000 kilos) de carbón, el cual es ingresado por tracción humana, el operador lo empuja y lleva hasta los sitios de tolva interno de la mina, donde se deposita el carbón (descargue). Esta tolva interna tiene una compuerta, la cual abre, llena el coche y hala hacia fuera. El nuevo sistema de transporte consiste en una locomotora que funciona con energía eléctrica y tiene la capacidad de halar 12 coches, cada uno con capacidad de 1500 kilos (tonelada y media).

Por tal razón, la presente propuesta de investigación se ha realizado con base en la necesidad detectada en la mejora de métodos y procesos de producción, las cuales deben ser destinadas a mejorar las líneas de procesos y sistemas de producción ya existentes, con vistas a aumentar los rendimientos y disminuir los costos de producción, así como la mejora de la calidad y la revalorización de la mina; la investigación se centra en el proceso del transporte interno en la mina de explotación subterránea de carbón, el shipio; se selecciona este proceso, ya que los costos de extracción de los minerales suelen aumentar a lo largo de la vida en las minas. Al extenderse las labores a profundidad hace mayores las distancias de transporte interno, generando un considerable aumento de contratación de personal para que continúen realizando un transporte artesanal por tracción humana y así mantener un flujo aceptable de extracción del mineral a superficie, esto crea condiciones más difíciles en cuanto al alto riesgo y vulnerabilidad al que está expuesto la empresa por diferentes variables de costos y posible accidentabilidad.

De igual manera, con este proyecto se contribuye académicamente a la implementación de conceptos financieros reales y prácticos que puede y deben ser asimilados en el gremio de la pequeña y mediana minería del carbón, ya que evidenciaría como la preparación y evaluación de proyectos se ha transformado en un instrumento de uso prioritario entre los agentes económicos que participan en cualquiera de las etapas de la asignación de recursos para implementar iniciativas de inversión [9]; la evaluación de este proyecto se encargará de construir toda la metodología necesaria para reducir al máximo cualquier posibilidad de pérdida financiera y contar con una base científica que sustente las inversiones a realizar, dicha metodología usara diversas herramientas matemáticas, para realizar los pronósticos necesarios, los cuales se basan en técnicas estadísticas entre las series de tiempo, la regresión lineal, el análisis por mínimos cuadrados, etc.. todo esto encaminado a dejar la consigna en el gremio minero de la región, de que los estudios de evaluación y de viabilidad de un proyecto minero deben ser prioritarios en sus organizaciones empresariales ya que abarcan un conjunto de actividades de investigación y desarrollo conducentes a definir y valorar el proyecto de manera total y/o parcial en sus diferentes aspectos 
técnicos y económicos, que nos facilitara el poder realizar toma de decisiones financieras con un planteamiento lógico de viabilidad demostrable.

\section{Metodología}

El presente trabajo de investigación se elaboró mediante un enfoque Interpretativo por cuanto se describieron las diversas situaciones financieras de la mina en estudio y sus finanzas para poder establecer una evaluación financiera, de la misma manera se desarrolló bajo un diseño de investigación documental ya que se trabajó con fuentes bibliográficas de la organización para poder estudiar y develar las condiciones actuales y las cifras para poder generar criterios acerca de la viabilidad de la implementación de la semimecanización del sistema de transporte interno de la mina Shipio.

\section{Desarrollo}

\section{Diagnóstico financiero del proceso de extracción y de trans- porte al interior de la mina Shipio.}

En la mina Shipio se realiza un tipo de minería subterránea que emplea un sistema de transporte artesanal, apoyado por un coche o vagoneta que depende de la tracción humana, para el desplazamiento del carbón mineral desde el interior de la mina hasta el sitio de descargue (tolva interna). A medida que se extienden las labores de profundidad, se hacen mayores las distancias de transporte interno, lo que requiere cada vez más de contratación de personal para mantener las labores de la mina. Adicionalmente, esto viene acompañado de un mayor riesgo y vulnerabilidad para la empresa por posible accidentabilidad y diferentes variables de costos.

En la tabla II se relacionan los costos actuales del sistema de transporte interno en la Mina Shipio.

Tabla II. Costos de transporte interno en la mina Shipio.

\begin{tabular}{|c|c|}
\hline Parámetros & Shipio \\
\hline Cantidad de cocheros & 14 \\
\hline Ciclos día & 19,5 \\
\hline Ton dia transportadas & 273 \\
\hline Ton mes & 6006 \\
\hline Salario básico mes & $\$ 1.500 .000$ \\
\hline Seguridad social $(40,96 \%)$ & $\$ 302.169$ \\
\hline Prestaciones sociales $(21,82 \%)$ & $\$ 327.300$ \\
\hline Salario mes & $\$ 2.129 .469$ \\
\hline Costo mes cocheros & $\$ 29.812 .564$ \\
\hline Costo año cocheros & $\$ 298.125 .644$ \\
\hline
\end{tabular}

Como se observa en la tabla 5, actualmente se encuentran contratados 14 cocheros que realizan 19,5 ciclos al día, transportando 273 toneladas de carbón diariamente (6006 mensuales). El salario mensual incluidas las prestaciones sociales y seguridad social de cada uno de estos cocheros, es de $\$ 2.129 .469$, lo que constituye un costo mensual de $\$ 29.812 .564$. Como la mina desarrolla labores durante diez meses, al año el costo total de transporte interno en la mina Shipio asciende a $\$ 298.125 .644$.

\section{Costos actuales de producción}

Los índices proyectados de la mina Shipio se encuentran en 6.000 toneladas mensuales siendo un valor anual de 60.000 toneladas, puesto que las proyecciones de producción de la mina se ubican en 6.000 toneladas al mes, que al año equivaldrían a 60.000 toneladas, ya que en esta mina se elabora solamente diez meses estos costos vienen dados por tonelada y están expresados de la siguiente manera, señalados en la tabla I.

Tabla III. Descripción de costos por tonelada de carbón

\begin{tabular}{|l|r|}
\hline Devengado & $\$ 30.000$ \\
\hline Seguridad social portonelada & $\$ 5.682$ \\
\hline $\begin{array}{l}\text { Prestaciones sociales por } \\
\text { tonelada }\end{array}$ & $\$ 9.000$ \\
\hline Consumo energia & $\$ 2.955$ \\
\hline A CPM & $\$ 409$ \\
\hline Ferreteria & $\$ 6.364$ \\
\hline Madera & $\$ 4.545$ \\
\hline Flete transporte & $\$ 9.500$ \\
\hline Explosivos & $\$ 1.000$ \\
\hline Transporte personal & $\$ 455$ \\
\hline Dotación & $\$ 1.515$ \\
\hline Administración & $\$ 2.000$ \\
\hline Servidumbre & $\$ 4.000$ \\
\hline Regalias & $\$ 4.800$ \\
\hline Total costos Ton & $\mathbf{\$ 8 2 . 2 2 4}$ \\
\hline Total Ton producidas/mes & $\mathbf{6 . 0 0 0}$ \\
\hline Total costos producción $/$ mes & $\mathbf{4 9 3 . 3 4 5 . 4 5 5}$ \\
\hline Total costos producción/anual & $\mathbf{\$ 4 . 9 3 3 . 4 5 4 . 5 4 5}$ \\
\hline Precio venta & $\mathbf{\$ 1 2 2 . 0 0 0}$ \\
\hline
\end{tabular}

En la tabla III se devela el costo para producir una tonelada de carbón siendo de $\$ 82.224$, traduciéndose a un costo mensual de $\$ 493.345 .455$, esto con una producción de 6.000 toneladas. En unos años los costos se elevan a $\$ 4.933 .454 .545$.

Si se tiene en cuenta la tonelada de carbón, esta se vende a un 
Carolina Marulanda-Ascanio, Ender José Barrientos-Monsalve, Karla Johana Sánchez-Mujica, Blanca Liliana Velázquez-Carrascal, Nubiola Ceballos-Leyva.

costo de $\$ 122.000$, y los ingresos mensuales superan los $\$ 732.000 .000$, y los anuales a 7.320.000.000. Es por ello que si se analiza la rentabilidad de este sistema va ser de $32,6 \%$ (P.V. - C.V./P.V), que corresponde a $\$ 2.386 .500 .000$.

\section{Proyecciones del personal (cocheros) de transporte interno de la mina}

Tabla IV. Personal para transporte interno mina el shipio proyección año 2017-2023

\begin{tabular}{|c|c|c|c|c|c|c|c|}
\hline Parámetros & Año 2017 & Año 2018 & Año 2019 & Año 2020 & Año 2021 & Año 2022 & Año 2023 \\
\hline Cantidad de cocheros & 14 & 21 & 28 & 41 & 52 & 63 & 70 \\
\hline $\begin{array}{c}\text { distancia recorrido } \\
\text { ciclo Mits }\end{array}$ & 1600 & 2000 & 2400 & 2800 & 3200 & 3600 & 4000 \\
\hline $\begin{array}{c}\text { Aproxi avance anual } \\
\text { distancias mts }\end{array}$ & 200 & 200 & 200 & 200 & 200 & 200 & 200 \\
\hline $\begin{array}{l}\text { Velocidad promedio } \\
\text { cochero mts /minutos }\end{array}$ & 65 & 65 & 65 & 65 & 65 & 65 & 65 \\
\hline $\begin{array}{c}\text { Tiempo de ciclo } \\
\text { minutos }\end{array}$ & 25 & 31 & 37 & 43 & 49 & 55 & 62 \\
\hline $\begin{array}{l}\text { horas } s \text { turno laboradas } \\
\text { cochero }\end{array}$ & 8 & 8 & 8 & 8 & 8 & 8 & 8 \\
\hline $\begin{array}{l}\text { Ciclos turno } \\
\end{array}$ & 19,5 & 15,6 & 13 & 11,1 & 9,8 & 8,7 & 7,8 \\
\hline Ton dia transportadas & 273 & 328 & 364 & 457 & 507 & 546 & 546 \\
\hline Ton mes & 6006 & 7207 & 8008 & 10051 & 11154 & 12012 & 12012 \\
\hline Salario básico mes & $\$ 1.500 .000$ & \begin{tabular}{|l|}
$\$ 1.552 .500$ \\
\end{tabular} & \begin{tabular}{|l|l|}
$\$ 1.608 .390$ \\
\end{tabular} & $\$ 1.663 .075$ & $\$ 1.716 .294$ & $\$ 1.771 .215$ & $\begin{array}{l}1.827 .894 \\
\end{array}$ \\
\hline $\begin{array}{c}\text { Seguridad social } \\
(40,96 \%)\end{array}$ & $\$ 302.169$ & $\$ 312.745$ & $\$ 324.004$ & $\$ 335.020$ & $\$ 345.740$ & $\$ 356.804$ & $\$ 368.222$ \\
\hline $\begin{array}{c}\text { Prestaciones sociales } \\
(21,82 \%)\end{array}$ & $\$ 327.300$ & $\$ 338.756$ & $\$ 350.951$ & $\$ 362.883$ & $\$ 374.495$ & $\$ 386.479$ & $\$ 398.846$ \\
\hline Salariomes & $\$ 2.129 .469$ & $\$ 2.204 .000$ & $\$ 2.283 .344$ & $\$ 2.360978$ & $\$ 2.436 .529$ & $\$ 2.514 .498$ & $\$ 2.594 .962$ \\
\hline $\begin{array}{l}\text { Costo mes cocheros } \\
\end{array}$ & $\$ 29.812 .564$ & $\$ 46.284 .006$ & \begin{tabular}{|l|}
$\$ 63.933 .641$ \\
\end{tabular} & $\$ 96.800 .098$ & $\$ 126.699 .524$ & \begin{tabular}{|l|}
$\$ 158.413 .389$ \\
\end{tabular} & $\$ 181.647353$ \\
\hline Costo año cocheros & $\$ 298.125 .644$ & $\$ 462.840 .062$ & $\$ 639.336 .405$ & $\$ 968.000 .985$ & $\$ 1.266 .995 .240$ & $\$ 1.584 .133 .895$ & $\$ 1.816 .473 .532$ \\
\hline
\end{tabular}

En la anterior tabla se muestran las proyecciones del personal para transporte interno de la mina Shipio durante el periodo 2017-2023, en esta se observa que a medida que se amplían las labores de profundidad (distancia recorrida de los ciclos mts), se debe aumentar la cantidad de cocheros empleados al pasar de 14 en 2017 a 70 en 2023, este incremento en el número de cocheros permite reducir el número de ciclos de turno en el periodo, que en 2017 eran 19,5 y en 2023 7,8. Por otra parte, el incremento en el número de cocheros se traduce en el doble de toneladas de carbón transportadas al día, al pasar de 273 toneladas transportadas a 546. De esta manera las toneladas del mineral transportado al mes, también crecen el doble en el periodo.

El salario mensual de cada uno de los cocheros en 2017 es de $\$ 2.129 .469$ incluidas las prestaciones sociales y seguridad social, constituyendo un costo mensual y anual por el total de cocheros de \$29.812.564 y \$298.125.644, respectivamente. Cabe notar, que el aumento del número de cocheros empleados incrementa cinco veces los costos anuales del transporte interno en el periodo analizado, pasando de \$298.125.644 en 2017 a $\$ 1.816 .473 .532$ en 2023.

\section{Costos totales e ingresos con el sistema de transporte interno tradicional (cocheros).}

La tabla IV describe los costos ingresos por tonelada de carbón, en ésta se observa que todos los costos aumentan a través del tiempo. El costo de la producción de una tonelada de carbón en el 2017 es de $\$ 82.224$, lo que se traduce en un costo mensual de $\$ 493.345 .455$, dada una producción al mes de 6.000 toneladas. $\mathrm{Al}$ año, los costos ascienden a $\$ 4.933 .454 .545$. En cuanto a los costos de producción del año 2023, el costo de la tonelada (\$142.595) de carbón incrementaría un 73\% con respecto al 2017, así como los costos mensuales $\$ 1.711 .140 .784$ con el doble de la producción de mineral (12.000 ton). En el periodo 2017-2013 los costos anules habrán aumentado $\$ 12.177 .907 .844$.

\section{Análisis de la viabilidad financiera del cambio de sistema de transporte interno en la mina Shipio}

Teniendo en cuenta que los costos del sistema de transporte interno de la mina Shipio son muy altos y llegan a superar a los ingresos con el paso del tiempo, se hace necesario mejorar este proceso mediante la implementación de un sistema semi-mecanizado, que consiste en una locomotora que funciona con energía eléctrica y tiene la capacidad de halar 12 coches, cada uno con capacidad de 1500 kilos (tonelada y media).

Tabla V. Caracterización adecuación metro de carrilera

\begin{tabular}{|c|c|c|c|c|}
\hline Insumos y/o servicios & Unidad & Cantidad & Vr. Unitario & Vr. Total \\
\hline Riel & Unidad & 467 & $\$ 220.000$ & $\$ 102.740 .000$ \\
\hline triturado 3/4 & $\mathrm{m} 3$ & 336 & $\$ 28.000$ & $\$ 9.408 .000$ \\
\hline Polin & Unidad & 2800 & $\$ 6.000$ & $\$ 16.800 .000$ \\
\hline Eclis as & Unidad & 934 & $\$ 6.000$ & $\$ 5.604 .000$ \\
\hline Clavo de bareta 4" & Cajas & 40 & $\$ 24.300$ & $\$ 972.000$ \\
\hline Mano de obra & metro & 1350 & $\$ 20.000$ & $\$ 27.000 .000$ \\
\hline \multicolumn{4}{|c|}{ Adecuación cruzada y niveles 1350 metros } & $\$ 162.524 .000$ \\
\hline \multicolumn{4}{|c|}{ Costo por metro adecuación carrilera } & $\$ 120.388$ \\
\hline
\end{tabular}

\section{Tipo de financiación de la Inversión}

La fuente de financiamiento de la inversión del sistema semi-mecanizado de transporte será la de endeudamiento con un banco comercial que ofrece una tasa efectiva mensual de $1,48 \%(19,28 \%$ E.A.) a un periodo de cuatro años con cuotas fijas mensuales. Según la tabla de amortización los pagos corresponderían a 48 cuotas mensuales de $\$ 11.027 .151$ más un seguro de vida de $\$ 433.550$ para un total de cuota más seguro de $\$ 11.460 .701$. Se estimó que los costos anuales de la financiación ascienden a $\$ 137.528 .413$ y el costo total del mismo a $\$ 550.113 .655$. Los demás costos se relacionan a continuación.

Tabla VI. Amortización de pagos del primer año

\begin{tabular}{|c|l|l|l|l|l|l|}
\hline Cuota & $\begin{array}{c}\text { Abono a } \\
\text { Interés }\end{array}$ & $\begin{array}{c}\text { Abono a } \\
\text { Capital }\end{array}$ & $\begin{array}{c}\text { Cuota Mensual } \\
\text { sin Seguros }\end{array}$ & $\begin{array}{c}\text { valor del } \\
\text { seguro de } \\
\text { vida asociado } \\
\text { a la Deuda }\end{array}$ & $\begin{array}{c}\text { cuota mensual } \\
\text { más seguro }\end{array}$ & saldo \\
\hline $\mathbf{0}$ & $\$$ & $\$$ & $\$$ & $\$ 433.550,00$ & $\$$ & $\$ 377.000 .000,00$ \\
\hline $\mathbf{1}$ & $\$ 5.579 .600,00$ & $\$ 5.447 .551,16$ & $\$ 11.027 .151,16$ & $\$ 433.550,00$ & $\$ 11.460 .701,16$ & $\$ 371.552 .448,84$ \\
\hline $\mathbf{2}$ & $\$ 5.498 .976,24$ & $\$ 5.528 .174,92$ & $\$ 11.027 .151,16$ & $\$ 433.550,00$ & $\$ 11.460 .701,16$ & $\$ 366.024 .273,92$ \\
\hline $\mathbf{3}$ & $\$ 5.417 .159,25$ & $\$ 5.609 .991,91$ & $\$ 11.027 .151,16$ & $\$ 433.550,00$ & $\$ 11.460 .701,16$ & $\$ 360.414 .282,02$ \\
\hline $\mathbf{4}$ & $\$ 5.334 .131,37$ & $\$ 5.693 .019,79$ & $\$ 11.027 .151,16$ & $\$ 433.550,00$ & $\$ 11.460 .701,16$ & $\$ 354.721 .262,23$ \\
\hline $\mathbf{5}$ & $\$ 5.249 .874,68$ & $\$ 5.777 .276,48$ & $\$ 11.027 .151,16$ & $\$ 433.550,00$ & $\$ 11.460 .701,16$ & $\$ 348.943 .985,75$ \\
\hline $\mathbf{6}$ & $\$ 5.164 .370,99$ & $\$ 5.862 .780,17$ & $\$ 11.027 .151,16$ & $\$ 433.550,00$ & $\$ 11.460 .701,16$ & $\$ 343.081 .205,58$ \\
\hline $\mathbf{7}$ & $\$ 5.077 .601,84$ & $\$ 5.949 .549,32$ & $\$ 11.027 .151,16$ & $\$ 433.550,00$ & $\$ 11.460 .701,16$ & $\$ 337.131 .656,26$ \\
\hline $\mathbf{8}$ & $\$ 4.989 .548,51$ & $\$ 6.037 .602,65$ & $\$ 11.027 .151,16$ & $\$ 433.550,00$ & $\$ 11.460 .701,16$ & $\$ 331.094 .053,62$ \\
\hline $\mathbf{9}$ & $\$ 4.900 .191,99$ & $\$ 6.126 .959,17$ & $\$ 11.027 .151,16$ & $\$ 433.550,00$ & $\$ 11.460 .701,16$ & $\$ 324.967 .094,45$ \\
\hline $\mathbf{1 0}$ & $\$ 4.809 .513,00$ & $\$ 6.217 .638,16$ & $\$ 11.027 .151,16$ & $\$ 433.550,00$ & $\$ 11.460 .701,16$ & $\$ 318.749 .456,29$ \\
\hline $\mathbf{1 1}$ & $\$ 4.717 .491,95$ & $\$ 6.309 .659,21$ & $\$ 11.027 .151,16$ & $\$ 433.550,00$ & $\$ 11.460 .701,16$ & $\$ 312.439 .797,08$ \\
\hline $\mathbf{1 2}$ & $\$ 4.624 .109,00$ & $\$ 6.403 .042,16$ & $\$ 11.027 .151,16$ & $\$ 433.550,00$ & $\$ 11.460 .701,16$ & $\$ 306.036 .754,92$ \\
\hline
\end{tabular}




\section{Descripción de Costos con operadores de locomotora}

Tabla VII. Relación de costos con operadores de locomotora

\begin{tabular}{|c|c|c|c|c|c|c|c|}
\hline Parámetros & Año 2017 & Año 2018 & Año 2019 & Año 2020 & Año 2021 & Año 2022 & Año 2023 \\
\hline Operadores de locomotora & 3 & 3 & 3 & 3 & 6 & 6 & 6 \\
\hline Distancia recorrido ciclo 0 Mts & 1600 & 2000 & 2400 & 2800 & 3200 & 3600 & 4000 \\
\hline $\begin{array}{l}\text { Aproxi avance anual distancias } \\
\text { mis }\end{array}$ & 200 & 200 & 200 & 200 & 200 & 200 & 200 \\
\hline $\begin{array}{l}\text { Velocidad promedio locomotora } \\
\text { mis / } / \text { minu tos }\end{array}$ & 220 & 220 & 220 & 220 & 220 & 220 & 220 \\
\hline Tiempo de ciclo minutos & 7 & 9 & 11 & 13 & 15 & 16 & 18 \\
\hline Coches de arrestre por ciclo & 12 & 12 & 12 & 12 & 12 & 12 & 12 \\
\hline Horas stu rmo laboradas operadores & 8 & 8 & 8 & 8 & 8 & 8 & 8 \\
\hline Ciclos por turno & 60 & 53 & 44 & 38 & 33 & 29 & 26 \\
\hline Ciclos de transporte requeridos & 23 & 27 & 30 & 38 & 42 & 46 & 46 \\
\hline Ton dia transportadas & 273 & 328 & 364 & 457 & 507 & 546 & 546 \\
\hline Ton mes & 6000 & 7000 & 8000 & 10000 & 11000 & 12000 & 12000 \\
\hline Salario básico mes & $\$ 1.500 .000$ & $\$ 1.552 .500$ & $\$ 1.608 .300$ & $\$ 1.663 .075$ & $\$ 1.716 .294$ & $\$ 1.771 .215$ & 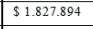 \\
\hline Seguridad social $(40,96 \%)$ & $\$ 302.169$ & $\$ 312.745$ & $\$ 324.004$ & $\$ 335.020$ & $\$ 345.740$ & $\$ 356.804$ & $\$ 368.222$ \\
\hline Prestaciones $(21,82 \%)$ & $\$ 327.300$ & $\$ 338.756$ & $\$ 350.951$ & $\$ 362.883$ & $\$ 374.495$ & 9386.479 & $\$ 398.846$ \\
\hline Salario mes & \begin{tabular}{|l|l|l|}
52.129 .469 \\
\end{tabular} & $\$ 2.204 .000$ & $\$ 2.283 .344$ & $\$ 2.360 .978$ & $\$ 2.436 .529$ & $\$ 2.514 .498$ & $\$ 2.594 .962$ \\
\hline Costo mes operadores & $\$ 6.388 .407$ & $\$ 6.612 .001$ & $\$ 6.850 .033$ & $\$ 7.082 .934$ & S14.619.176 & $\$ 15.086 .989$ & $\$ 15.569 .773$ \\
\hline Costo año operadores & $\$ 63.884 .066$ & $\$ 66.120 .009$ & $\$ 68.500 .329$ & $\$ 70.829340$ & $\$ 146.191 .758$ & $\$ 150.869 .805$ & $\$ 155.697 .731$ \\
\hline
\end{tabular}

En la anterior tabla se muestran las proyecciones del personal con el sistema de transporte semi-mecanizado durante el periodo 2017-2023, a diferencia del sistema de transporte tradicional, en éste la cantidad de operadores de locomotora empleados es más bajo, 3 en 2017 y 6 en 2023. El salario mensual de cada uno de los operadores en 2017 es de \$2.129.469 incluidas las prestaciones sociales y seguridad social, constituyendo un costo mensual y anual por el total de operadores de $\$ 6.388 .407$ y $\$ 63.884 .066$, respectivamente. Con este sistema los costos anuales de los trabajadores de las máquinas se reducen en \$234.241.577 en el primer año, puesto sólo que representan el $27 \%$ de los costos tradicionales. Cabe resaltar la gran diferencia en costos que se da al finalizar el periodo, esta diferencia equivale a $\$ 1.660 .775 .801$ puesto que los costos del sistema semi-mecanizado sólo representan en este periodo el 9\% de los tradicionales.

Tabla VIII. Costos totales por año, con búsqueda de recursos de apalancamiento para efectuar la inversión del proyecto

\begin{tabular}{|c|c|c|c|c|c|c|c|}
\hline Mina & $\mathbf{2 0 1 7}$ & $\mathbf{2 0 1 8}$ & $\mathbf{2 0 1 9}$ & $\mathbf{2 0 2 0}$ & $\mathbf{2 0 2 1}$ & $\mathbf{2 0 2 2}$ & $\mathbf{2 0 2 3}$ \\
\hline $\begin{array}{c}\text { Cuotas anuales Préstamo } \\
\text { Inversión de locomotora }\end{array}$ & $\$ 137.528 .414$ & $\$ 137.528 .414$ & $\$ 137.528 .414$ & $\$ 137.094 .864$ & $\$ 0$ & $\$ 0$ & $\$ 0$ \\
\hline Salarios anual & $\$ 63.884 .066$ & $\$ 66.122 .009$ & $\$ 68.500 .329$ & $\$ 70.829 .340$ & $\$ 146.191 .758$ & $\$ 150.869 .895$ & $\$ 155.697 .731$ \\
\hline Mantenimiento locomotora & $\$ 14.500 .000$ & $\$ 15.225 .000$ & $\$ 15.986 .250$ & $\$ 16.785563$ & $\$ 17.624 .841$ & $\$ 18.506 .083$ & $\$ 19.431 .387$ \\
\hline Ootal costo año & $\$ 215.912 .480$ & $\$ 218.873 .423$ & $\$ 222.014 .993$ & $\$ 224.709 .767$ & $\$ 163.816 .599$ & $\$ 169.375 .977$ & $\$ 175.129 .118$ \\
\hline
\end{tabular}

La anterior tabla presenta los costos totales del sistema de transporte interno semi-mecanizado, éstos son más representativos hasta el año 2020, ya que en este periodo se termina de cancelar la inversión, después empiezan a descender, al pasar de \$215.912.480 en 2017 a \$175.129.118 en 2023.

\section{Descripción de costos de tonelada de carbón con operadores de locomotora}

Tabla IX. Costos por tonelada de carbón del sistema semi-mecanizado

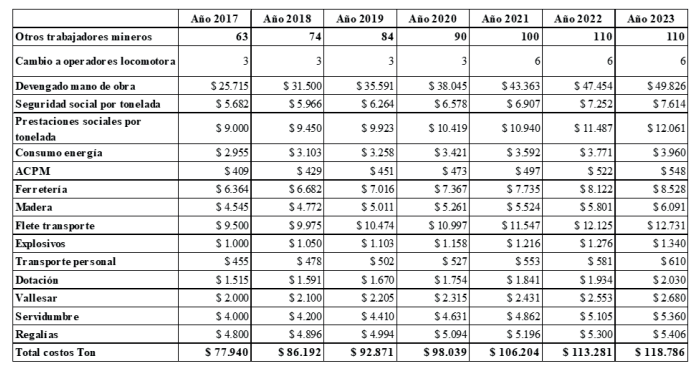

Por su parte, los costos por tonelada de carbón empiezan a reducirse dado el decremento en costos del sistema de transporte, para el 2017 una tonelada de carbón equivaldría a $\$ 77.940$, $\$ 4.285$ menos que en el sistema tradicional. Llegando a reducirse hasta en $\$ 23.809$ el costo en tonelada de carbón en el año 2023 .

Tabla X. Descripción de costos e ingresos anuales por tonelada de carbón del sistema semi-mecanizado

\begin{tabular}{|c|c|c|c|c|c|c|c|}
\hline & 2017 & 2018 & 2019 & 2020 & 2021 & 2022 & 2023 \\
\hline Total costos Ion & $\$ 77.940$ & $\$ 86.192$ & $\$ 92.871$ & $\$ 98.039$ & $\$ 100.204$ & $\$ 113281$ & $\$ 118.786$ \\
\hline $\begin{array}{l}\text { al Ton } \\
\text { al Ton }\end{array}$ & 6.000 & 7.000 & 8.000 & 10.000 & 11.000 & 12.000 & 12.000 \\
\hline \begin{tabular}{|l} 
producidasmines \\
Total costos
\end{tabular} & $\$ 467.640 .000$ & 5603.345 .750 & $\$ 742.966200$ & $\$ 980388.140$ & $\$ 1.168 .243 .541$ & $\$ 1.359 .371 .291$ & $\$ 1.425 .432 .004$ \\
\hline \begin{tabular}{|l} 
Protact costos \\
produccion/anual \\
parcial
\end{tabular} & $\$ 4.676 .400 .000$ & S 6.033.457.500 & 57.429 .662 .600 & 50.803 .881 .403 & $\$ 11.682 .435 .410$ & $\$ 13.593 .712 .915$ & S 14.25..320.044 \\
\hline 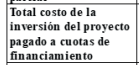 & $\$ 137.528 .414$ & $\$ 137.528 .414$ & $\$ 137.528 .414$ & $\$ 137.094 .864$ & so & so & so \\
\hline $\begin{array}{l}\text { Total costo anual } \\
\text { mant tenimiento del } \\
\text { proverto }\end{array}$ & $\$ 14.500 .000$ & s15.225.000 & $\$ 15.986250$ & S 16.785.563 & $\$ 17.624 .841$ & $\$ 18.506 .083$ & $\$ 19.431 .387$ \\
\hline \begin{tabular}{|l|}
$\begin{array}{l}\text { Total costos } \\
\text { produccion/anual nnalal }\end{array}$ \\
\end{tabular} & $\$ 4.828 .428 .414$ & S 6.186.210.914 & \$ 7.583.177.264 & $\$ 9.957 .761 .829$ & $\$ 11.700 .060 .251$ & $\$ 13.612 .218 .997$ & S 14.273.751.431 \\
\hline Precio ve, & $\$ 122.000$ & 8123.830 & $\$ 125.687$ & $\$ 127.573$ & $\$ 129.486$ & $\$ 131.429$ & $\$ 133.400$ \\
\hline Total precio de & $\$ 732.000 .000$ & $\$ 866.810 .000$ & $\$ 1.005 .499 .600$ & $\$ 1.275 .727 .618$ & $\$ 1.424 .349 .885$ & $\$ 1.577 .143 .782$ & $\$ 1.60$ \\
\hline \begin{tabular}{|l} 
Total precio \\
\end{tabular} & $\$ 7.320 .000 .000$ & $\$ 8.668 .100 .000$ & $\$ 10.054 .9960000$ & $\$ 12.757276 .175$ & $\$ 14.243 .498 .849$ & $\$ 15.771 .437 .817$ & $\$ 16.008 .009384$ \\
\hline Rentabilidad & $0.04 \%$ & $28,63 \%$ & ,58\% & $21,94 \%$ & $17,86 \%$ & $13,69 \%$ & $10,83 \%$ \\
\hline Rentabilidad COP & $\$ 2.491 .571 .586$ & S2.481.889.086 & $\$ 2.471 .818 .736$ & \$2.799.514.346 & \$2.543.438.599 & 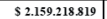 & $\$ 1.734 .257 .953$ \\
\hline
\end{tabular}

En la tabla XV se realiza un comparativo de los costos totales de la producción del sistema de transporte semi-mecanizado con los ingresos percibidos en el periodo 2017-2023. Los costos totales anuales de producción son más bajos que los del sistema tradicional, haciéndose más significativa la diferencia a través del tiempo, para el 2017 esta diferencia equivale a $\$ 105.071 .586$ y al 2023 ascendería a \$2.837.656.413 en costos. Lo anterior permite que la rentabilidad de este sistema sea de 34\% en 2017 (P.V. - C.V./P.V), que equivale a \$2.491.571.586 (1,4\% por encima del tradicional). Contrario a las rentabilidades que incluyen el sistema tradicional de transporte en sus costos, las del sistema semi-mecanizado son positivas en todo el periodo, 2017-\$ 2.491.571.586, 2018-\$ 2.481.889.086, 2019-\$ 2.471.818.736, 2020-\$ 2.799.514.346, 2021-\$ 2.543.438.599, 2022-\$2.159.218.819 у 2023-\$ 1.734.257.953.

\section{Análisis de eficiencia del sistema semi-mecanizado}

Para realizar el análisis de eficiencia del sistema de semi-mecanización del transporte interno, se requiere establecer el flujo de caja de efectivo del periodo analizado, tal como lo muestra la tabla XIV, compuesta de siete periodos proyectados anualmente.

Tabla XI. Datos de flujo de caja

\begin{tabular}{|c|r|}
\hline DATOS & VALORES \\
\hline Numero de periodos & 7 \\
\hline Tipo de periodos & anual \\
\hline Tasa de descuento & $19,28 \%$ \\
\hline
\end{tabular}

Con una inversión de $\$ 377.000 .000$ en el sistema de transporte interno, [10] el valor presente Neto del proyecto arroja un saldo positivo (mayor que cero), es decir, que éste maximizaría la inversión inicial en \$4.711.248.180 a una tasa de descuento del 19,28\% (esta tasa de descuento corresponde a la tasa de interés vigente del mercado). A su vez, la Tasa Interna de Retorno del proyecto (TIR) es mayor que cero (376\%), la implementación de este sistema devuelve aproximadamente cuatro veces 
el capital invertido más una ganancia adicional, el valor de esta TIR está explicada por la rentabilidad que ofrecen los proyectos mineros, por lo que el proyecto debe ejecutarse.

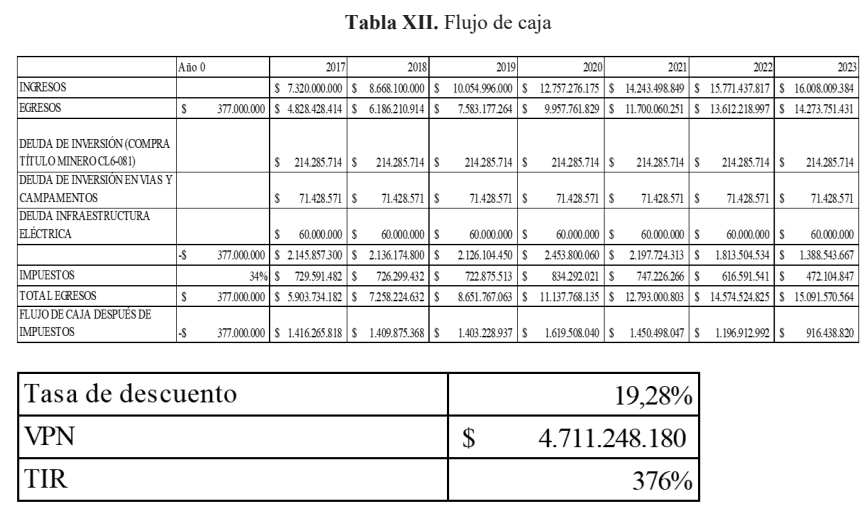

Tabla XIII. Relación Costo Beneficio

\begin{tabular}{|c|c|}
\hline VPN INGRESOS & $\$ 40.491 .019 .837,83$ \\
\hline VPN EGRESOS & $\$ 5.465 .248 .179,94$ \\
\hline B/C & 7,4 \\
\hline
\end{tabular}

El costo beneficio consiste en integrar el valor presente neto de éstos se procede a realizar el cociente entre ellos, [11] siendo la relación costo-beneficio del sistema semi-mecanizado del transporte del carbón es de 7,4 cuando el B/C es mayor que 1, este valor de beneficios es mayor a los costos del proyecto es por ello que se acepta el proyecto y se hace la recomendación de hacer la inversión por los beneficios que conlleva este sistema a la mina, teniendo una ganancia de 7,4 unidades monetarias adicionales es por ello lo atractivo del proyecto.

\section{Conclusiones}

Para el trabajo de investigación realizado se determinó la factibilidad financiera para la de la semimecanización del método de transporte interno de la mina Shipio, 2017 a través de un análisis costo beneficio con el fin de establecer la conveniencia de la ejecución del mismo.

El uso y explotación del sistema de semimecanización (locomotora) para el desarrollo del presente trabajo fueron de $\$ 377.000 .000$, inversión que recuperaría en el primer año de la implementación.

El estudio se realizó a siete años porque el contrato de concesión de venta del carbón con Termotasajero S.A. E.S.P. vence en este tiempo, los resultados del análisis financiero arrojaron un VPN de \$4.711.248.180, TIR de 376\% y una relación B/C de 7,4 .

El valor de la TIR obedece a los siguientes parámetros: La adquisición de un título minero es un buen negocio en el que las cuantiosas inversiones requeridas dejan grandes utilidades, puesto que el mineral se ofrece en forma natural y no lleva ningún proceso de beneficio. Además, el valor de las reservas va aumentando con el tiempo por lo que lo hace más atractivo.

\section{Recomendaciones}

Los resultados del análisis financiero del proyecto recomiendan a la directiva de la empresa Carbones de Toledo S.A., realizar las inversiones requeridas para el desarrollo de la implementación de la semimecanización del sistema de transporte interno de la mina Shipio, ya que el contrato de venta con la empresa Termotasajero S.A. E.S.P., asegura la rentabilidad del proyecto y las proyecciones a futuro tanto en producción y venta del producto, dan una suficiente garantía para cubrir los costos de la inversión y además recuperar con creces el capital invertido.

Es por ello la importancia de la publicación y socialización del presente trabajo para tener en cuenta los aportes financieros que la semimecanización en la mina puede llegar, teniendo bondades a futuro para el sector económico de la región, y asi ayudar a impulsar por medio de las investigaciones a los directivos de la mina en sumar en la inversión que a futuro los beneficios serán considerables.

\section{Bibliografía}

[1] M. d. j. Zambrano, «Indice de desempeño exportador del carbon en Norte de Santander,» Espacios, vol. 38, $\mathrm{n}^{\circ} \mathrm{http}: / / \mathrm{re}-$ vistaespacios.com/a17v38/a17v38n38p03.pdf., p. 3, 2017.

[2] D. N. d. planeación, «Agenda interna para la productividad,» informe, $\mathrm{n}^{\mathrm{o}} \mathrm{http}: / / \mathrm{www}$.mincit.gov.co/loader.php?1Servi-

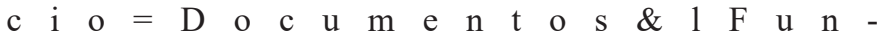
cion $=$ verPdf\&id $=23225 \&$ name $=$ InsumosNortedeSantander.pd f\&prefijo=file el 01 de mayo del 2017, 2007.

[3] D. n. d. Planeación, «Agenda interna para la productividad,» informe anual, $\mathrm{n}^{\mathrm{o}} \mathrm{http}: / / \mathrm{www}$.mincit.gov.co/loader.php?1$\mathrm{S}$ e $\mathrm{r}$ vi c i o $=\mathrm{D}$ o c u m e $\mathrm{nto}$ s \& $1 \mathrm{~F} \mathrm{u} \mathrm{n} \mathrm{-}$ cion $=$ verPdf $\& i d=23225 \&$ name $=$ InsumosNortedeSantander.pd f\&prefijo=file el 01 de mayo del 2017, 2007.

[4] R. N. A. Barrientos Monsalve E, «Modelo gerencial dentro del paradigma del capital intelectual para el sector hotelero de la ciudad de Cúcuta-Colombia,» Revista Argentina de Investigación en negocios, vol. 4, $\mathrm{n}^{\circ}$ recuperado de: http://ppct.caicyt.gov.ar/index.php/rain/article/view/V4n1a7/pdf, pp. 85-95, 2018.

[5] R. n. N. R. S. J. B. R. M. Barrientos monsalve E, «La teoría fundamentada como herramienta para investigar los escenarios del marketing,» Mundo fesc, vol. 8, $\mathrm{n}^{\circ} 16$. Recuperado de: http://www.fesc.edu.co/Revistas/OJS/index.php/mundofesc/article/view/307/345, pp. 95-99, 2018.

[6] M. J., Evaluación Financiera de proyectos, Ecoe.https://www.ecoediciones.com/wp-content/u- 
ploads/2017/04/Evaluaci $\%$ C3\%B3n-financiera-de-proyectos4ta-Edici\%C3\%B3n.pdf, 2007.

[7] m. G. J. P. O. L. Jaimes Mora S, «Factores Psicosociales que Influyen en el Comportamiento Laboral de Acuerdo con los Procesos de Gestión Administrativa,» Mundo Fesc, vol. 5, nº 9. recuperado de : https://dialnet.unirioja.es/servlet/articulo?codi$\mathrm{go}_{\mathrm{o}}=5286658$, pp. 64-68, 2015.

[8] R. N. A. Barrientos Monsalve E, «FACTORES PSICOSOCIALES QUE INFLUYEN EN EL COMPORTAMIENTO LABORAL DE ACUERDO CON LOS PROCESOS DE GESTIÓN ADMINISTRATIVA Y DEL TALENTO HUMANO QUE PRESENTAN LOS EMPLEADOS DE LA EMPRESA DISTRAVES S.A DE CÚCUTA,» Mundo Fesc, vol. 5, no 10. recuperado de: http://www.fesc.edu.co/Revistas/OJS/index.php/mundofesc/article/view/73, pp. 23-26, 2015.

[9] J. C. L. C. E. R. Hernandez Suarez C, «Modelos de aplicación de ecuaciones diferenciales de primer orden con geogebra,» Mundo Fesc, $\mathrm{n}^{\circ}$ 11. recuperadop de: https://dialnet.unirioja.es/servlet/articulo?codigo=5627639, pp. 7-15, 2016.

[10] B. C. Nidia, «Familia: un estado del arte en grupos de investigación de Colciencias en psicología entre 2010-2015,» mundo fesc, pp. 33-47, 2016.

[11] L. G. Fredy, «S.O.S. SE VIENEN LAS NIIF,» mundo fesc, vol. 3, no 5.recuperado de: http://www.fesc.edu.co/Revistas/OJS/index.php/mundofesc/article/view/34, pp. 29-32, 2013. 